\title{
KNOT ENERGIES AND KNOT INVARIANTS
}

\author{
XIAO-SONG LiN \\ Department of Mathematics \\ University of California \\ Riverside, CA 92521 \\ xl@math.ucr.edu \\ To record what has happened, ancient people tie knots. \\ - I ching, the Chinese classic of 1027-771 B.C.
}

Knots are fascinating objects. When fastening a rope, the distinction between a knot and a "slip-knot" (one that can be undone by pulling) must be recognized very early in human history. We even developed a subconscious about knots: When we are puzzled or troubled, we have a feeling of being knotted somewhere. The mathematical study of knots started much later though. It was inspired in the middle of the nineteenth century by the vortex theory of fluid dynamics (see [11] for a vivid description of this history). The development of modern topology in the first half of the twentieth century provided a solid background for a mathematical theory of knots. Yet we only began to see the full scope of knot theory in the last decade, starting with the discovery of the Jones polynomial in 1984 (see [13] for a survey of the history of knot theory up to Jones' discovery). In 1989, Witten generalized the Jones polynomial using his Chern-Simons path integral. Finally, in 1990-92, the development of knot theory culminated in the theory of Vassiliev knot invariants, which provides probably the most general framework for the study of the combinatorics of knots. Through the study of Vassiliev knot invariants, we see that although the abundance of knots in varieties is distinctively visible, this abundance does not come from any randomness. The combinatorics of knots embraces almost all fundamental symmetries of mathematics and physics that we know. Such a pervasive nature is not common among topological and geometric objects mathematicians are in favor of. For the reader's convenience, we have collected several excellent expository papers on these developments in the references (see $[1,2,4,8,14])$.

Geometers are restless in their effort of searching for geometric objects with "maximal homogeneity". Here, of course, the measurement of homogeneity are different in different situations. Actually, it is the key point to recognize in a given geometric setting what should be the measurement of homogeneity. Thus, in classical Riemannian geometry, we know that various curvatures are the key measurement of homogeneity; we measure length or area for immersions of circles and surfaces into a Riemannian manifold and developed the theories of geodesics and minimal surfaces; in gauge theory, we study connections minimizing the Yang-Mills 
etc.. And there is always the moduli problem if geometric objects with maximal homogeneity are not unique. So, we may also ask for smooth imbeddings $S^{1} \rightarrow \mathbb{R}^{3}$, which we will refer to as geometric knots, with the "most perfect" shape among all geometric knots isotopic to each other. This geometric side of knot theory is much less mature than the combinatorial side of knot theory. It seems to be not completely clear yet as of what should be the most fundamental measurement for the homogeneity of a geometric knot within its isotopy class. One of the purposes of this article is then to argue that such measurements of homogeneity satisfying the criteria set forth in the foundational paper of Freedman, He and Wang [6] may not be unique. There seems to be a spectrum of Möbius knot energies related with functionals on geometric knots appeared in integral formulae of Vassiliev knot invariants coming from perturbative expansion of Witten's Chern-Simons path integral (they are called Gauss functionals). Of course, unless we could understand the dynamical behavior of geometric knots with respect to these Möbius energies, their nature remains to be a mystery.

Classically, functionals on loop spaces people have studied include the length functional and holonomy functionals. Functionals defined only on embedded curves have caught people's attention lately, to a large part due to the recent advance in knot theory. The elementary discussion on those Gauss functionals on geometric knots in this article reminds us classical integral geometry where different measurements on the same geometric object are shown to be related. Hopefully, this will motivate further interesting in geometric knot theory.

We would like to thank Colin Adams for the invitation of writing an article for this special issue of Chaos, Solitons and Fractals. We have talked about the topics of this article in geometry/topology seminars at UCD, UCR and UCSD. It is a pleasure to thank the participants of these seminars, in particular, Professors Mike Freedman and Oleg Viro, for their interests and comments. It would be impossible to have the work presented here done without continuing discussions with Zhenghan Wang. We appreciate very much the help and stimulation we get from him.

\section{$\S 1$. Möbius energies}

We define a geometric knot to be a smooth embedding $\gamma: S^{1} \rightarrow \mathbb{R}^{3}$. Here, the oriented circle $S^{1}$ comes with no particular parameterization.

Two geometric knots $\gamma_{1}$ and $\gamma_{2}$ are equivalent (or isotopic) if there is an orientation preserving diffeomorphism $\rho: \mathbb{R}^{3} \rightarrow \mathbb{R}^{3}$ such that $\gamma_{2}=\rho \gamma_{1}$. A knot is simply an equivalence class of geometric knots. See Figure 1. So, a geometric knot is unknotted if it can be deformed to a round circle without passing through itself.

The Möbius energy $E(\gamma)$ of a geometric knot $\gamma$, defined in [6], is given as follows: Suppose $S^{1}$ is parameterized by $u$ and $d u$ is the positive volume form of $S^{1}$ coming from that parameterization. For $u, v \in S^{1}, u \neq v$, denote by $D(\gamma(v), \gamma(u))$ be the minimum of the lengths of sub-arcs of $\gamma$ from $\gamma(u)$ to $\gamma(v)$. Then

$$
E(\gamma)=\int_{u \in S^{1}}|\dot{\gamma}(u)| d u\left(\int_{v \in S^{1}, v \neq u}\left\{\frac{1}{|\gamma(v)-\gamma(u)|^{2}}-\frac{1}{D(\gamma(v), \gamma(u))^{2}}\right\}|\dot{\gamma}(v)| d v\right)
$$

The integral is independent of the parameterization of $S^{1}$. It is therefore a positive 

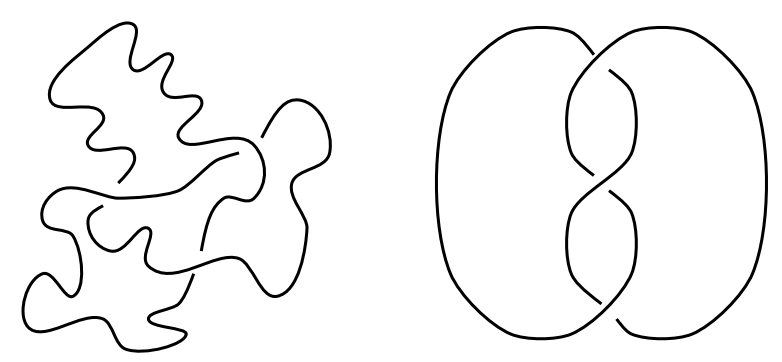

Figure 1. Two equivalent (isotopic) geometric knot.

The idea is that as a geometric knot $\gamma$ deforms and tends to acquire a double point, $E(\gamma)$ will blow up and thus constraints the deformation of $\gamma$ within its isotopy class. Ideally, every geometric knot would deform to a unique energy-minimizing geometric knot within its isotopy class via the gradient flow of $E$ (the direction where $E$ is decreasing). Although this is not true in general, we will see below that geometric knots with smaller $E$ do look more "homogeneous". See [9] for a table of many energy-minimizing geometric knots obtained by computer simulation.

A fundamental property of the energy functional $E$ discovered in [6] is its invariance under Möbius transformation of $\mathbb{R}^{3} \cup\{\infty\}$. Möbius transformations of $\mathbb{R}^{3} \cup\{\infty\}$ are the 10-dimensional Lie group of angle-preserving diffeomorphisms of $\mathbb{R}^{3} \cup\{\infty\}$ generated by inversion in 2-spheres. The Möbius invariance of $E$ says that if $T$ is a Möbius transformation and $T \gamma \subset \mathbb{R}^{3}$, then $E(T \gamma)=E(\gamma)$. If $T \gamma \subset \mathbb{R}^{3} \cup\{\infty\}$, one may generalize the energy functional $E$ to infinite curves in $\mathbb{R}^{3}$ and then we have $E\left(T \gamma \cap \mathbb{R}^{3}\right)=E(\gamma)-4$. See Theorem 2.1 of $[6]$.

The Möbius invariance of $E$ led to its reformulation by P. Doyle and O. Schramm in terms of some conformally invariant data.

Let $u, v \in S^{1}, u \neq v$. In $\mathbb{R}^{3}$, there are exactly two round, oriented circles or oriented straight lines $U_{v}$ and $V_{u}$ passing through $\gamma(u)$ and $\gamma(v)$ and tangent to $\dot{\gamma}(u)$ at $\gamma(u)$ and $\dot{\gamma}(v)$ at $\gamma(v)$ respectively. Let $\alpha=\alpha(u, v)$ be the angle between $U_{u}$ and $V_{u}, 0 \leq \alpha \leq \pi$. Define

$$
E_{\cos }(\gamma)=\int_{u \in S^{1}}|\dot{\gamma}(u)| d u\left(\int_{v \in S^{1}, v \neq u} \frac{1-\cos \alpha}{|\gamma(v)-\gamma(u)|^{2}}|\dot{\gamma}(v)| d v\right)
$$

The Möbius invariance of $E_{\cos }$ is more or less transparent.

The functional $E_{\cos }$ may be interpreted in terms of "excess lengths". Fix $u \in S^{1}$ and we may assume that $\gamma(u)=0$ and $\dot{\gamma}(u)$ is horizontal. We apply the Möbius inversion $x \mapsto \frac{x}{|x|^{2}}$ about the unit 2-sphere centered at the origin to $\gamma$. Then $\gamma$ becomes an asymptotically horizontal infinite curve $\gamma_{\infty}$ in $\mathbb{R}^{3}$. The Möbius inversion sends each $U_{v}$ to a horizontal straight line $L_{v}$ and they are all parallel to each other for different $v$ 's. The angle $\alpha$ between $U_{v}$ and $V_{u}$ is the same as the angle between $\dot{\gamma}_{\infty}(v)$ and $L_{v}$. Although both $\gamma_{\infty}$ and a horizontal straight line have infinite lengths, these two infinities are comparable in the sense that their difference can be made finite. This excess length can be computed in the following way.

Let $s$ be the arc length parameter of $\gamma_{\infty}$. Then, from $s_{1}$ to $s_{2}$, the horizontal distance one travels along $\gamma_{\infty}$ is

$$
\int^{s_{2}} \cos \alpha d s
$$


Therefore the horizontal excess length of $\gamma_{\infty}$ is

$$
\int_{\gamma_{\infty}}(1-\cos \alpha) d s<\infty
$$

But

$$
d s=\left|\dot{\gamma}_{\infty}(v)\right| d v \quad \text { and } \quad \gamma_{\infty}(v)=\frac{\gamma(v)}{|\gamma(v)|^{2}} .
$$

Moreover, simple vector calculus shows

$$
\left|\dot{\gamma}_{\infty}(v)\right|=\frac{|\dot{\gamma}(v)|}{|\gamma(v)|^{2}}
$$

Thus, the first integration in $E_{\text {cos }}$ is exactly the horizontal excess length of $\gamma_{\infty}$. From this interpretation, it is quite clear that a geometric knot which has a "highly oscillated segment" can not be a $E_{\mathrm{cos}^{-}}$minimizing geometric knot.

Proposition. (Doyle-Schramm) $E=E_{\mathrm{cos}}+4$.

There is one more Möbius energy paired with $E_{\mathrm{cos}}$ :

$$
E_{\sin }(\gamma)=\int_{u \in S^{1}}|\dot{\gamma}(u)| d u\left(\int_{v \in S^{1}, v \neq u} \frac{\sin \alpha}{|\gamma(v)-\gamma(u)|^{2}}|\dot{\gamma}(v)| d v\right)
$$

The energy $E_{\text {sin }}$ lacks the smoothness of $E_{\text {cos }}$. To see this, let $\alpha$ be the angle between two unit vectors $\mathbf{v}_{1}, \mathbf{v}_{2} \in S^{2}, 0 \leq \alpha \leq \pi$. Then

$$
1-\cos \alpha=1-\mathbf{v}_{1} \cdot \mathbf{v}_{2}
$$

is a smooth function on $S^{2} \times S^{2}$, whereas

$$
\sin \alpha=\left|\mathbf{v}_{1} \times \mathbf{v}_{2}\right|
$$

is not a smooth function on $S^{2} \times S^{2}$.

Similar to the excess length interpretation of $E_{\mathrm{cos}}$, the first integration in $E_{\sin }$, which is equal to

$$
\int_{\gamma_{\infty}} \sin \alpha d s<\infty
$$

can be interpreted as the "total momentum" of $\gamma_{\infty}$ with respect to its asymptotic direction. Again, to minimize $E_{\text {sin }}, \gamma$ should not have "highly oscillated" segments.

\section{$\S 2$. Gauss functionals}

We define chord diagrams first, which are originated in the study of Vassiliev knot invariants. A chord diagram with $n$ chords consists of $2 n$ distinct points on $S^{1}$ which are paired into $n$ pairs. We stick a chord to each paired points to indicate the pairing. Chord diagrams are combinatorial objects so that two chord diagrams are 
of $S^{1}$ sending pairs to pairs. A Gauss diagram is a chord diagram whose end points of chords are ordered in consistence with the orientation of $S^{1}$. See Figure 2.

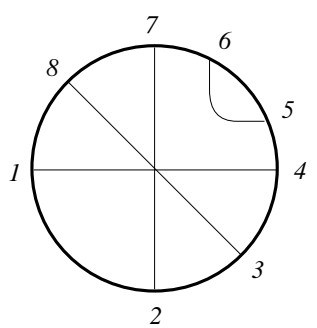

Figure 2. A Gauss diagram.

We will denote by $C_{k}$ the configuration space of $k$ ordered distinct points on $S^{1}$ such that the ordering of points is consistent with the orientation of $S^{1}$. It is an oriented open $k$-dimensional manifold.

Let $\omega$ be the standard volume form of the unit 2 -sphere $S^{2}$ such that

$$
\int_{S^{2}} \omega=1
$$

For $x \in \mathbb{R}^{3} \backslash\{0\}$, we will denote by $\omega(x)$ the pull-back of $\omega$ under the map

$$
\mathbb{R}^{3} \backslash\{0\} \rightarrow S^{2}: x \mapsto \frac{x}{|x|}
$$

We have

$$
\omega(x)=\frac{1}{8 \pi} \frac{<x, d x, d x>}{|x|^{3}}=\frac{1}{4 \pi} \frac{x_{1} d x_{2} d x_{3}+x_{2} d x_{3} d x_{1}+x_{3} d x_{1} d x_{2}}{|x|^{3}}
$$

where $<,,>$ is the mixed product in $\mathbb{R}^{3}$.

Let $\gamma$ be a geometric knot and $D$ be a Gauss diagram with $n$ chords. We may define a functional $I_{D}(\gamma)$ as follows. First, we order the chords in $D$. This allows us to define a map

$$
F_{D}: C_{2 n} \longrightarrow\left(S^{2}\right)^{n}
$$

For $\left(u_{1}, u_{2}, \ldots, u_{2 n}\right) \in C_{2 n}$, the $j$-th coordinate of its image in $\left(S^{2}\right)^{n}$ under $F_{D}$ is

$$
\frac{\gamma\left(u_{i^{\prime}}\right)-\gamma\left(u_{i}\right)}{\left|\gamma\left(u_{i^{\prime}}\right)-\gamma\left(u_{i}\right)\right|}
$$

if $i$ and $i^{\prime}$ are paired in $D$ as the $j$-th pair and $i<i^{\prime}$. Then

$$
\mathcal{I}_{D}(\gamma)=\int_{C_{2 n}} F_{D}^{*}\left(\omega^{n}\right)
$$

Obviously, $\mathcal{I}_{D}$ is independent of the choice of orderings of chords in $D$ as 2 -forms commute. We will call $\mathcal{I}_{D}$ a Gauss functional on geometric knots.

We may also have a reduced Gauss functional $\overline{\mathcal{I}}_{D}$ associated with each chord 
chords. The cyclic group $\mathbb{Z}_{2 n}$ acts on the set of Gauss diagram having the same underlying chord diagram as that of $D$ by permuting end points of $D$ cyclicly. Then, by definition,

$$
\overline{\mathcal{I}}_{D}=\frac{1}{2 n} \sum_{\sigma \in \mathbb{Z}_{2 n}} \mathcal{I}_{\sigma D}
$$

Of course, if $\sigma D=D$ as Gauss diagrams for each $\sigma \in \mathbb{Z}_{2 n}$, we have $\overline{\mathcal{I}}_{D}=\mathcal{I}_{D}$.

If $D$ has only one chord, $\mathcal{I}_{D}(\gamma)$ is the classical writhe integral:

$$
\mathcal{I}_{w}(\gamma)=\frac{1}{4 \pi} \int_{C_{2}} \frac{<\gamma\left(u_{2}\right)-\gamma\left(u_{1}\right), \dot{\gamma}\left(u_{1}\right), \dot{\gamma}\left(u_{2}\right)>}{\left|\gamma\left(u_{2}\right)-\gamma\left(u_{1}\right)\right|^{3}} d u_{1} d u_{2}
$$

originated from Gauss' formula for the linking number of two disjoint geometric knots in the 3-space (a link).

Suppose $D$ has two chords which cross each other. We call such a chord diagram $X$. We have

$$
\begin{aligned}
\mathcal{I}_{X}(\gamma)=\left(\frac{1}{4 \pi}\right)^{2} \int_{C_{4}} & \frac{\left\langle\gamma\left(u_{3}\right)-\gamma\left(u_{1}\right), \dot{\gamma}\left(u_{1}\right), \dot{\gamma}\left(u_{3}\right)>\right.}{\left|\gamma\left(u_{3}\right)-\gamma\left(u_{1}\right)\right|^{3}} \\
& \frac{\left\langle\gamma\left(u_{4}\right)-\gamma\left(u_{2}\right), \dot{\gamma}\left(u_{2}\right), \dot{\gamma}\left(u_{4}\right)>\right.}{\left|\gamma\left(u_{4}\right)-\gamma\left(u_{2}\right)\right|^{3}} d u_{1} d u_{3} d u_{2} d u_{4} .
\end{aligned}
$$

Gauss functionals contain topological information of knots. When geometric knots are deformed within their isotopy classes, Gauss functionals will change their values. But the variation of Gauss functionals may be compensated by the variation of other different kinds of functionals on geometric knots so that together they form knot invariants: functionals having the same value on isotopic geometric knots. The classical case is the so-called Călugăreanu-Pohl self-linking formula expressing the self-linking number of a geometric knot with nowhere vanishing curvature as the sum of its writhe and total torsion [12]. Originated from the perturbative expansion of Witten's Chern-Simons path integral, modern generalizations of the self-linking formula form a vast family of knot invariants, possibly exhausts all Vassiliev knot invariants. See $[3,5]$.

In [10], the simplest generalization of the self-linking formula was studied in details. It involves the Gauss functional $\mathcal{I}_{X}$ and another functional $\mathcal{I}_{Y}$. To define $\mathcal{I}_{Y}$, let

$$
C_{3}(\gamma)=\left\{\left(u_{1}, u_{2}, u_{3}, x\right) ;\left(u_{1}, u_{2}, u_{3}\right) \in C_{3}, x \in \mathbb{R}^{3} \backslash\left\{\gamma\left(u_{1}\right), \gamma\left(u_{2}\right), \gamma\left(u_{3}\right)\right\}\right\}
$$

Also, denote

$$
H(u, x)=\frac{(\gamma(u)-x) \times \dot{\gamma}(u)}{|\gamma(u)-x|^{3}}
$$

For $x$ not on $\gamma$. Then

$$
\mathcal{I}_{Y}=\left(\frac{1}{\Lambda_{1}}\right)^{3} \int \quad<H\left(u_{1}, x\right), H\left(u_{2}, x\right), H\left(u_{3}, x\right)>d^{3} x d u_{1} d u_{2} d u_{3}
$$


The functional

$$
\frac{1}{4} \mathcal{I}_{X}-\frac{1}{3} \mathcal{I}_{Y}+\frac{1}{24}
$$

turns out to be an integer valued knot invariant which can be identified as the second coefficient of the Conway knot polynomial.

\section{$\S$ 3. The $X$-crossing number}

Let $D$ be a Gauss diagram with $n$ chords. For a geometric knot $\gamma$. Define

$$
\mathcal{C}_{D}(\gamma)=\int_{C_{2 n}}\left|F_{D}^{*}\left(\omega^{n}\right)\right|
$$

Here, if $F_{D}^{*}\left(\omega^{n}\right)=\lambda d_{\mathrm{vol}},\left|F_{D}^{*}\left(\omega^{n}\right)\right|=|\lambda| d_{\mathrm{vol}}$.

If $D$ is a chord diagram with $n$ chords, then define

$$
\overline{\mathcal{C}}_{D}=\frac{1}{2 n} \sum_{\sigma \in \mathbb{Z}_{2 n}} \mathcal{C}_{\sigma D}
$$

We will see that $\overline{\mathcal{C}}_{D}$ has the combinatorial meaning of being the "average $D$-crossing number". Here, some digression about the functionals $\overline{\mathcal{I}}_{w}=\mathcal{I}_{w}$ and $\overline{\mathcal{C}}_{w}=\mathcal{C}_{w}$ seems to be appropriate before we could straighten out the general case.

Using a partition of unit, the 2-form $\omega$ on $S^{2}$ can be decomposed as a sum of many 2 -forms supported in small neighborhoods of single points. Let $\omega_{\mathbf{v}}$ be one of these 2-forms supported in a small neighborhood of $\mathbf{v} \in S^{2}$ and $P_{\mathbf{v}}: \mathbb{R}^{3} \rightarrow \mathbb{R}^{2}$ be the orthogonal projection in the direction $\mathbf{v}$. If we replace $\omega$ by $\omega_{\mathbf{v}}$ in the functional $\mathcal{I}_{w}(\gamma)$ and $\mathcal{C}_{w}(\gamma)$, what we get, roughly speaking, are the algebraic crossing number $w(\gamma ; \mathbf{v})$ and the crossing number $n(\gamma ; \mathbf{v})$ of the plane projection $P_{\mathbf{v}}(\gamma)$, respectively. To be more precise,

$$
n(\gamma ; \mathbf{v})=\frac{1}{2} \sharp\left\{\left(u_{1}, u_{2}\right) \in C_{2} ; P_{\mathbf{v}}(\gamma)\left(u_{1}\right)=P_{\mathbf{v}}(\gamma)\left(u_{2}\right)\right\}
$$

and $w(\gamma ; \mathbf{v})$ represents a similar signed counting. The following proposition should therefore be quite clear (see [7] and [6]).

Proposition. We have

$$
\mathcal{I}_{w}(\gamma)=\frac{1}{4 \pi} \int_{\mathbf{v} \in S^{2}} w(\gamma ; \mathbf{v}) d_{\mathrm{vol}}(\mathbf{v})
$$

and

$$
\mathcal{C}_{w}(\gamma)=\frac{1}{4 \pi} \int_{\mathbf{v} \in S^{2}} n(\gamma ; \mathbf{v}) d_{\mathrm{vol}}(\mathbf{v})
$$

where $d_{\mathrm{vol}}$ is the volume element of $S^{2}$.

Therefore, $\overline{\mathcal{C}}_{w}(\gamma)$ (so as $\overline{\mathcal{I}}_{w}(\gamma)$ ) is the average, over all possible directions, of 
directions. Notice that we are looking at $\gamma$ "with one eye" in each direction. But $\gamma$ is in the 3-space. To get a more stereo-scopic image, we'd better to look at it with two or more eyes. This is actually the principle behind stereo-photography. With $\overline{\mathcal{C}}_{D}$ for a general chord diagram $D$, it seems that we are doing the same thing as in stereo-photography: First look at $\gamma$ through many individual eyes, and then try to combine the images obtained individually together in a certain way. The resulting picture is of course more complete (and more complicated) than what we get by looking at $\gamma$ with one eye.

Consider now the $X$ diagram. Let $\gamma$ be a geometric knot and $\mathbf{v}_{1}, \mathbf{v}_{2} \in S^{2}$. We define a number $n\left(\gamma ; \mathbf{v}_{1}, \mathbf{v}_{2}\right)$ as follows.

We first notice that there is a subset of $S^{2} \times S^{2}$ of full measure with the following property: If $\left(\mathbf{v}_{1}, \mathbf{v}_{2}\right)$ is in this subset, and $u_{1}, u_{2}, u_{3}, u_{4} \in S^{1}$ such that

$$
P_{\mathbf{v}_{1}}(\gamma)\left(u_{1}\right)=P_{\mathbf{v}_{1}}(\gamma)\left(u_{3}\right) \quad \text { and } \quad P_{\mathbf{v}_{2}}(\gamma)\left(u_{2}\right)=P_{\mathbf{v}_{2}}(\gamma)\left(u_{4}\right)
$$

then $u_{1}, u_{2}, u_{3}, u_{4}$ are distinct. With this said, we define

$$
\begin{array}{r}
n\left(\gamma ; \mathbf{v}_{1}, \mathbf{v}_{2}\right)=\frac{1}{4} \sharp\left\{\left(u_{1}, u_{2}, u_{3}, u_{4}\right) \in C_{4} ; P_{\mathbf{v}_{1}}(\gamma)\left(u_{1}\right)=P_{\mathbf{v}_{1}}(\gamma)\left(u_{3}\right),\right. \\
\left.P_{\mathbf{v}_{1}}(\gamma)\left(u_{2}\right)=P_{\mathbf{v}_{1}}(\gamma)\left(u_{4}\right)\right\}
\end{array}
$$

for $\left(\mathbf{v}_{1}, \mathbf{v}_{2}\right)$ in that subset of full measure.

A similar argument as in the previous proposition can be used to prove the following proposition.

Proposition. We have

$$
\mathcal{C}_{X}(\gamma)=\left(\frac{1}{4 \pi}\right)^{2} \iint_{S^{2} \times S^{2}} n\left(\gamma ; \mathbf{v}_{1}, \mathbf{v}_{2}\right) d_{\mathrm{vol}}\left(\mathbf{v}_{1}\right) d_{\mathrm{vol}}\left(\mathbf{v}_{2}\right)
$$

The crossing number of a knot is defined to be the minimum of crossing numbers of regular plane projections of that knot, where a plane projection of a knot is called regular if it has only transverse double pints and the number of double points is the crossing number of that regular plane projection.

Denote by $[\gamma]$ the knot type of a geometric knot $\gamma$, and by $C([\gamma])$ the crossing number of the knot $[\gamma]$. Then we have

$$
C([\gamma]) \leq \mathcal{C}_{w}(\gamma)
$$

We would like to have a similar lower bound depending only on the knot type $[\gamma]$ for $\mathcal{C}_{X}(\gamma)$.

Suppose we have a plane curve with only transverse double points as its singular points. It is given by an immersion $S^{1} \rightarrow \mathbb{R}^{2}$. The preimages of the transverse double points are paired such that points in a pair have the same image. This gives 


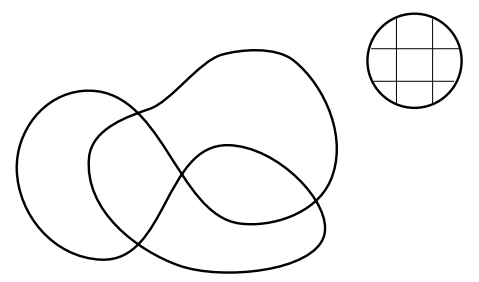

Figure 3. A plane curve and its chord diagram.

For example, if $K$ is a knot which has the plane curve in Figure 3 as one of its regular projection, then $C_{X}(K) \leq 4$.

Definition. The $X$-crossing number $C_{X}$ of a knot is the minimum of numbers of pairs of intersecting chords in chord diagrams of regular projections of that knot.

Theorem. Let $\gamma$ be a geometric knot. We have

$$
C_{X}([\gamma]) \leq \frac{1}{2} \mathcal{C}_{X}(\gamma)
$$

Proof (a sketch). The proof is based on the previous proposition and the fact that $\mathcal{C}_{X}$ is scalar invariant. Fix a vector $\mathbf{v}_{1}$ such that $P_{\mathbf{v}_{1}}(\gamma)$ is regular. Since $\mathcal{C}_{X}$ is scalar invariant, we may assume that the preimage on $\gamma$ of the two intersecting segments of $P_{\mathbf{v}_{1}}(\gamma)$ at a double points are very close to each other. Then, roughly speaking, for almost all $\mathbf{v}_{2}$, near the double points of $P_{\mathbf{v}_{1}}(\gamma)$, we see $\gamma$ in the direction $\mathbf{v}_{2}$ as much as in the direction $\mathbf{v}_{1}$. We may even see more in the direction $\mathbf{v}_{2}$, of course. Therefore,

$$
2 C_{X}([\gamma]) \leq n\left(\gamma ; \mathbf{v}_{1}, \mathbf{v}_{2}\right)
$$

where the factor 2 is because we don't distinguish $\mathbf{v}_{1}$ and $\mathbf{v}_{2}$ in the counting of $C_{X}$. This, together with the previous proposition, proves the theorem.

In general, for any chord diagram $D$, we may define the $D$-crossing number $C_{D}$ of a knot and state similar theorems about $C_{D}$ and $\overline{\mathcal{C}}_{D}$. We will not get into the details of such generalizations since they are quite straightforward. But we do want to address one thing about $D$-crossing numbers unknown to the author.

A chord diagram $D$ is called a sub-diagram of another chord diagram $D^{\prime}$ if $D$ can be obtained from $D^{\prime}$ by dropping off some chords. The $D$-crossing number of a knot is the minimum of numbers of $D$ 's as sub-diagrams of chord diagrams of regular projections of the given knot. The usual crossing number is the $D$-crossing number with $D$ being the chord diagram having one chord. It is an easy fact that the usual crossing number bounds the number of knots.

Proposition. The $X$-crossing number $C_{X}$ bounds the number of knots. In other words, given a positive number $N$, there are only finite many knots with $C_{X} \leq N$.

The proof is very simple. Just note that the only way to get infinitely many chord diagrams with bounded numbers of $X$ sub-diagrams is to start with a finite 
ones. Chord diagrams obtained in such a way cannot be chord diagrams of regular projections of new knots.

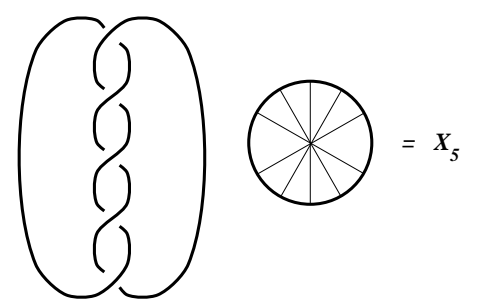

Figure 4. A regular projection of $K_{5}$ and its chord diagram.

Let $X_{n}$ be the chord diagram with $n$ chords such that every pair of chords intersect each other. For each odd $n \geq 3$, we have a knot $K_{n}$ (the so-called $(2, n)$ torus knot) which has a regular projection with $X_{n}$ as its chord diagram. See Figure 4. Notice that the only sub-diagrams of $X_{n}$ are $X_{k}, k \leq n$. Therefore, if $D$ is a chord diagram with $n$ chords and not equal to $X_{n}$, the $D$-crossing number can not bound the number of knots. Moreover, as pointed out by Zhenghan Wang, the family of twist knots in Figure 5 shows that the $X_{n}$-crossing number, $n \geq 3$, can neither bound the number of knots. But it is still possible that $X_{n}$-crossing numbers could be used to control the number of knots in some sense. It is known that the number of knots grows at most like an exponential function of the crossing number $C$. In fact, it is shown in [6] that the number of knots with energy $E \leq M$ is at most $\simeq(0.264)(1.658)^{M}$. We say that a $D$-crossing number controls the number of knots if the number of knots with bounded $D$-crossing numbers grows like a polynomial function of the crossing number. More specifically, we ask the following question.

Question. Does the number of knots with bounded $X_{n}$-crossing numbers grows like the function $C^{n-2}$ of the crossing number $C$ ?
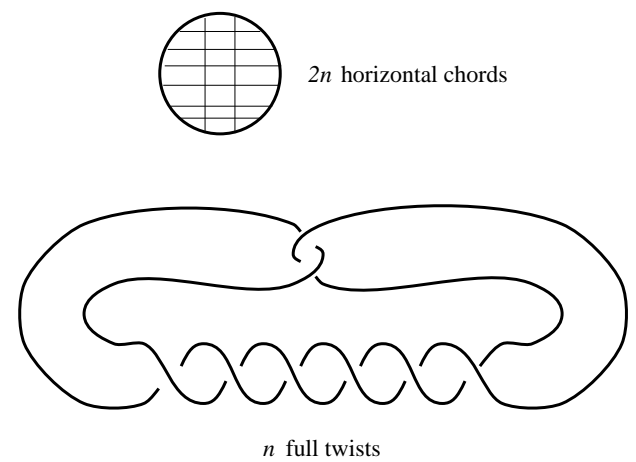

Figure 5. Twist knots and their chord diagrams.

\section{§. Möbius $X$-energies}

Here again, we will treat the chord diagram $X$ only and there is no difficult to consider general chord diagrams.

Let $\gamma$ be a geometric knot. For $\left(u_{1}, u_{2}, u_{3}, u_{4}\right) \in C_{4}$, as in $\S 1$, let $\alpha_{13}$ be the 
$\gamma\left(u_{2}\right), \gamma\left(u_{4}\right), \dot{\gamma}\left(u_{2}\right), \dot{\gamma}\left(u_{4}\right)$. We define

$$
E_{\mathrm{cos}, X}(\gamma)=\int_{C_{4}} \frac{1-\cos \alpha_{13}}{\left|\gamma\left(u_{3}\right)-\gamma\left(u_{1}\right)\right|^{2}} \frac{1-\cos \alpha_{24}}{\left|\gamma\left(u_{4}\right)-\gamma\left(u_{2}\right)\right|^{2}} d_{\mathrm{vol}}\left(u_{1}\right) d_{\mathrm{vol}}\left(u_{2}\right) d_{\mathrm{vol}}\left(u_{3}\right) d_{\mathrm{vol}}\left(u_{4}\right)
$$

and

$$
E_{\mathrm{sin}, X}(\gamma)=\int_{C_{4}} \frac{\sin \alpha_{13}}{\left|\gamma\left(u_{3}\right)-\gamma\left(u_{1}\right)\right|^{2}} \frac{\sin \alpha_{24}}{\left|\gamma\left(u_{4}\right)-\gamma\left(u_{2}\right)\right|^{2}} d_{\mathrm{vol}}\left(u_{1}\right) d_{\mathrm{vol}}\left(u_{2}\right) d_{\mathrm{vol}}\left(u_{3}\right) d_{\mathrm{vol}}\left(u_{4}\right)
$$

where $d_{\mathrm{vol}}$ is the volume element of $\gamma$.

Following [6], we consider the following properties as essential to qualify a functional on geometric knots being a Möbius energy functional:

(1) it is a non-negative functional and equals to zero iff on a round circle;

(2) it is invariant under Möbius transformation; and

(3) it bounds the number of knots.

Remark: According to the discussion of $\S 3$, we probably should modify (3) to ask only that general energies functionals control the number of knots.

The functional $E_{\cos }$ and $E_{\text {sin }}$ obviously satisfy (1) and (2). The proof that (3) holds for $E_{\cos }$ is not easy. It depends on two main results in [6] that $E$ is Möbius invariant and bounds the number of knots. We will see below that it is much easier to prove that $E_{\text {sin }}$ bounds the number of knots.

The properties (1) and (2) hold obviously for the functional $E_{\cos , X}$ and $E_{\sin , X}$. We have the following theorem.

Theorem. Let $\gamma$ be a geometric knot. Then

$$
C([\gamma]) \leq \frac{1}{4 \pi} E_{\sin }(\gamma)
$$

and

$$
C_{X}([\gamma]) \leq \frac{1}{2}\left(\frac{1}{4 \pi}\right)^{2} E_{\sin , X}(\gamma)
$$

Therefore, both $E_{\mathrm{sin}}$ and $E_{\mathrm{sin}, X}$ bound the number of knots.

Proof. The proof relies on some simple vector calculus.

Suppose we have there unit vectors $\mathbf{u}, \mathbf{v}$ and $\mathbf{w}$ in $\mathbb{R}^{3}$. Consider $\mathbf{w}$ as a segment with two vectors $\mathbf{u}$ and $\mathbf{v}$ stuck to its initial and terminal points respectively. We may repeat the construction in $\S 1$ to get an angle $\alpha$ out of this setting, $0 \leq \alpha \leq \pi$. If $\mathbf{u}$ and $\mathbf{w}$ are not collinear, we may let $\mathbf{u}^{\prime}$ be reflection of $\mathbf{u}$ with respect to $\mathbf{w}$ in the plane spanned by $\mathbf{u}$ and $\mathbf{w}$. Then $\alpha$ is the angle between $\mathbf{u}^{\prime}$ and $\mathbf{v}$. We may calculate

$$
\mathbf{u}^{\prime}=2(\mathbf{u} \cdot \mathbf{w}) \mathbf{w}-\mathbf{u}
$$

So, in particular, we have 
Thus,

$$
|<\mathbf{w}, \mathbf{u}, \mathbf{v}>| \leq \sin \alpha .
$$

This inequality still holds if $\mathbf{u}$ and $\mathbf{w}$ are collinear.

For $u, v \in S^{1}$, use the above inequality, we have

$$
\frac{|<\gamma(v)-\gamma(u), \dot{\gamma}(u), \dot{\gamma}(v)>|}{|\gamma(v)-\gamma(u)|^{3}} \leq \frac{\sin \alpha}{|\gamma(v)-\gamma(u)|^{2}}|\dot{\gamma}(u)||\dot{\gamma}(v)| \text {. }
$$

This implies the inequalities in the theorem. Therefor, both $E_{\sin }$ and $E_{\sin , X}$ bound the number of knots.

As

$$
\sin \alpha \sim \alpha \quad \text { and } \quad 1-\cos \alpha \sim \frac{\alpha^{2}}{2}
$$

when $\alpha$ is small, energies involving cosine appear to be "smaller" than those involving sine, at least locally. We don't know whether they are all compatible to each other.

Question. Does $E_{\mathrm{cos}, X}$ bound the number of knots?

Question. Could we interpret $E_{\cos , X}$ as measuring a certain kind of excess areas?

\section{REFERENCES}

1. Arnold, V. I., The Vassiliev theory of discriminants and knots, Proceedings of First European Congress of Mathematics, Prog. Math. vol. 119, Birkhauser, Basel, 1994.

2. Bar-Natan, D, On the Vassiliev knot invariants, Topology 34 (1995), 423-472.

3. Perturbative Chern-Simons theory, J. Knot Theory Rami. 4 (1995), 503-547.

4. Birman, J., New points of view in knot theory, Bull. Amer. Math. Soc. (N.S.) 28 (1993), $253-287$.

5. Bott, R. and Taubes, C., On the self-linking of knots, J. Math. Phys. 35 (1994), 5247-5287.

6. Freedman, M., He, Z.-X. and Wang, Z., Möbius energy of knots and unknots, Ann. Math. 139 (1994), 1-50.

7. Fuller, F., The writhing number of a space curve, Proc. Natl. Acad. Sci. USA 68 (1971), 815-819.

8. Kauffman, L., Functional integration and the theory of knots, J. Math. Phys. 36 (1995), 2402-2429.

9. Kusner, R. and Sullivan, J., Möbius energies for knots and links, surfaces and submanifolds, Proceedings of 1993 Georgia International Topology Conference (to appear).

10. Lin, X.-S. and Wang, Z., Integral geometry of plane curves and knot invariants, J. Diff. Geom. (to appear).

11. Lomonaco, S., Jr., The modern legacies of Thomson's atomic vortex theory in classical electrodynamics, The Interface of Knots and Physics (L. Kauffman, ed.), Proc. Symp. Appl. Math. vol. 51, Amer. Math. Soc., Providence, 1996. 
13. Przytycki, J., History of the knot theory from Vandermonde to Jones, Proceedings of XXIVth National Congress of the Mexican Mathematical Society, Soc. Mat. Mexicana, Mexico City, 1992.

14. Vassiliev, V. A., Invariants of knots and complements of discriminants, Developments in Mathematics: the Moscow School, Chapman \& Hall, London, 1993. 